\title{
Current efforts towards safe and effective live attenuated vaccines against African swine fever: challenges and prospects
}

Tao Wang ${ }^{1}$, Rui Luo ${ }^{1,2}$, Yuan Sun ${ }^{1}$ and Hua-Ji Qiu' ${ }^{1,2^{*}}$ (1)

\begin{abstract}
Background: African swine fever (ASF) is a fatal hemorrhagic disease in domestic pigs and wild boar caused by African swine fever virus (ASFV). Since ASF has been introduced into Europe and Asia, the major pig-raising areas, posing a huge threat to the pork industry worldwide. Currently, prevention and control of ASF are basically dependent on strict biosecurity measures and stamping-out policy once ASF occurs.

Main text: The major risks of ASF spread are insufficient biosecurity measures and human behaviors. Therefore, a safe and effective vaccine seems to be a reasonable demand for the prevention and control of ASF. Due to the efficacy advantage over other types of vaccines, live attenuated vaccines (LAVs), especially virulence-associated genes deleted vaccines, are likely to be put into emergency and conditional use in restricted areas if ASF is out of control in a country with a huge pig population and pork consumption, like China. However, the safety, efficacy, and genetic stability of current candidate ASF LAVs require comprehensive clinical evaluations prior to country-wide field application. Several critical issues need to be addressed to commercialize an ideal ASF LAV, including a stable cell line for manufacturing vaccines, differentiation of infected from vaccinated animals (DIVA), and cross-protection from different genotypes.
\end{abstract}

Conclusion: A safe and effective DIVA vaccine and an accompanying diagnostic assay will facilitate the prevention, control, and eradication of ASF, which is quite challenging in the near future.

Keywords: African swine fever, Live attenuated vaccine, Efficacy, Safety, Differentiation of infected from vaccinated animals

\footnotetext{
*Correspondence: qiuhuaji@caas.cn

1 State Key Laboratory of Veterinary Biotechnology, Harbin Veterinary

Research Institute, Chinese Academy of Agricultural Sciences,

Harbin 150069, China

Full list of author information is available at the end of the article
}

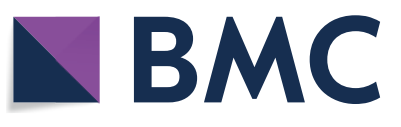

Aur(s) 2021. Open Access This article is licensed under a Creative Commons Attribution 4.0 International License, which permits use, sharing, adaptation, distribution and reproduction in any medium or format, as long as you give appropriate credit to the original author(s) and the source, provide a link to the Creative Commons licence, and indicate if changes were made. The images or other third party material in this article are included in the article's Creative Commons licence, unless indicated otherwise in a credit line to the material. If material is not included in the article's Creative Commons licence and your intended use is not permitted by statutory regulation or exceeds the permitted use, you will need to obtain permission directly from the copyright holder. To view a copy of this licence, visit http://creativecommons.org/licenses/by/4.0/. The Creative Commons Public Domain Dedication waiver (http://creativeco mmons.org/publicdomain/zero/1.0/) applies to the data made available in this article, unless otherwise stated in a credit line to the data. 


\section{Graphical Abstract}

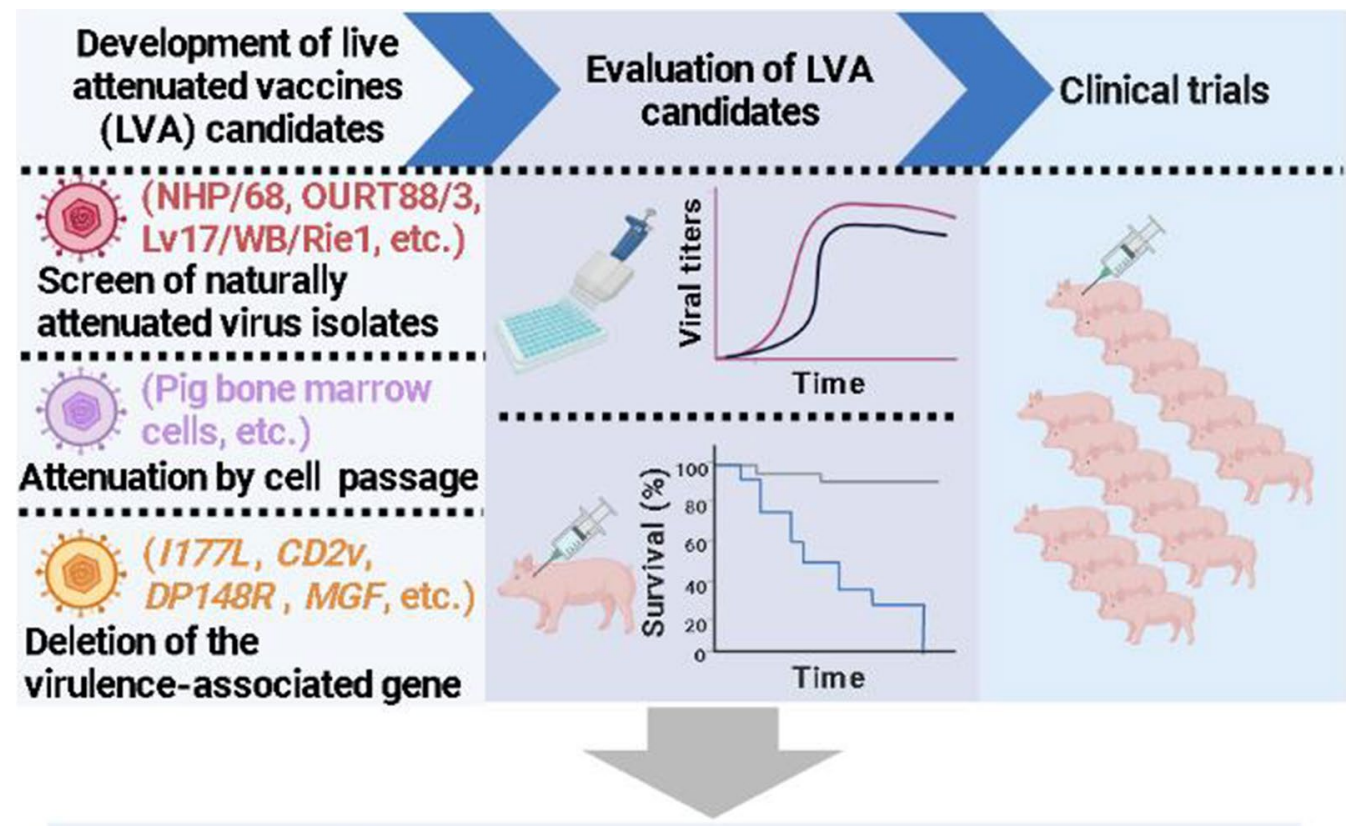

Major challenges impeding LVA development

1. Differentiation of infected from vaccinated animals

2. Selection of cell lines for vaccine production

3. Enhancement of cross-protection

\section{Background}

African swine fever (ASF) is a widespread hemorrhagic and fatal disease caused by ASF virus (ASFV) that occurs in domestic pigs and wild boar [1]. ASF was first discovered in 1921 in Kenya, and it has been endemic in many countries in Africa [2]. In 1957, ASF was introduced for the first time to the Iberian Peninsula and had circulated for more than 30 years. The disease severely struck the local pig industry and was not eradicated until 1996 (except for Sardinia) through biosecurity measures and eradication programs. In 2007, ASF was reintroduced from Africa to Europe (the Caucasus), spreading quickly to most Eastern European countries. The situation was worsened considerably as ASF spread to China in 2018 and quickly spread to 11 Asian countries [3]. Moreover, ASF spread to some European Union member states, including Belgium, the Czech Republic, Romania, Bulgaria, and Germany. Since no commercial vaccine is currently available, the prevention and control of ASF are essentially based on biosecurity and rapid test-removal policy. The pandemic of ASF has caused significant economic losses and threatened global pork production.
ASFV is a member of the family Asfarviridae (African swine fever and related viruses) and is the only known DNA arbovirus $[1,5]$. The ASFV genome is a doublestranded DNA molecule of 170-194 kb that contains 150-167 open reading frames (ORF), depending on the virus strains $[1,4]$. Based on the nucleotide differences at the end of the B646L (p72) gene, 24 ASFV genotypes have been identified [5]. The lack of safe and effective ASF vaccines is a big gap in the prevention and control of ASF [5]. Compared with other types of ASF vaccines, live attenuated vaccines (LAVs) can provide complete homologous and partial heterologous protection, representing a promising and feasible vaccine strategy in the foreseeable future [6]. With further safety improvement, LAVs are likely to be put into emergency and conditional use in restricted areas if ASF emerges or reemerges or is out of control in a country with a huge pig population and pork consumption, like China and the USA. 


\section{Strategies for generating ASF LAV candidates Screen of naturally attenuated virus isolates}

Naturally attenuated (NA) strains with reduced virulence and/or non-hemadsorbing (non-HAD) will occur during the natural epidemic of ASFV [7-11]. Notably, the non-HAD genotype I ASFV strains NH/P68 and OUR T88/3 were isolated from chronically infected pigs and soft ticks, respectively, when ASFV was circulating in the Iberian Peninsula [7, 8]. Pigs that survived infection with the NH/P68, or OUR T88/3, can protect against the virulent ASFV strain infection, indicating that the NA ASFV strain has the potential to be developed as a LAV. However, some side effects were also reported, including necrotic skin lesions and the swelling of joints, emphasizing that NA ASFV strains have residual virulence. Gallardo et al. isolated a non-HAD genotype II NA ASFV strain, Lv17/WB/Rie1, from wild boar in 2017 [10]. It can protect wild boar from challenge with the virulent ASFV Arm07 strain [10, 11]. Nevertheless, the safety, virus shedding, and genetic stability of Lv17/WB/Rie1 need to be further evaluated. Two years after the ASF outbreak in China, low virulent ASFV strains were isolated in 2020 [12]. Due to residual virulence, the NA strains are unlikely to be developed as LAVs. Further deletion of virulence-associated genes (VAG) based on NA candidates may improve the safety of LAVs.

\section{Attenuation by cell passage}

In addition to NA ASFV isolates, the continuous passage of a virulent ASFV strain in heterologous cells or hosts is a feasible strategy to develop LAVs. When ASF was first introduced into Europe, the researchers found that the virulent ASFV strains were attenuated after the continuous passage in porcine bone marrow or kidney cells $[13,14]$. Subsequent challenge experiments showed that the pigs immunized with the attenuated strain were protected against virulent strain infection. Although the attenuated strains showed a great promise in laboratory evaluation, the results of the field trials were disappointing [13]. Using a similar strategy, the virulent ASFV strain Stavropol 01/08 was attenuated by passages in a hybrid cell line $\mathrm{A}_{4} \mathrm{C}_{2} / 9 \mathrm{k}$. The ASFV of passages 24 and 33 in $\mathrm{A}_{4} \mathrm{C}_{2} / 9 \mathrm{k}$ cells and passage 20 in CV-1 cells lost pathogenicity to pigs but did not protect pigs against virulent strain infection [15]. The virulent genotype II ASFV Georgia strain (ASFV-G) lost pathogenicity and immunogenicity after 110 passages in Vero cells [16]. As mentioned above, the immunogenicity and efficacy of the cell-adapted LAVs differ significantly and need to be evaluated in pigs.

A recent study reported that the ASF vaccine strain ASFV-G- $\Delta \mathrm{I} 177 \mathrm{~L} / \Delta \mathrm{LVR}$, which was generated by adaptation of the ASFV-G- $\triangle$ I177L strain to the porcine fetal kidney cell line (PIPEC), displayed attenuation and protective efficacy similar to ASFV-G- $\Delta$ I177L [17]. Although this discovery brings hope to the large-scale production of the ASF vaccine in cell lines, the safety and genetic stability of the ASFV-G- $\Delta$ I177L/ $\Delta$ LVR strain still needs further evaluation. Furthermore, we recently demonstrated that ASFV adapted to HEK293T cells with deletion of the left-end variable MGF genes [18]. The deletion of MGF300 $(1 L, 2 R$, and $4 L)$ and MGF360 $(8 L, 9 L, 10 L$, and $11 \mathrm{~L}$ ) genes might be important for the adaptation of ASFV to HEK293T cells at the early stage [18]. Moreover, the pathogenicity and immunogenicity of the HEK293T cell-adapted ASFV will be evaluated in pigs. In the future, the NA ASFV strains or VAG deleted attenuated vaccine candidate strains can be adapted to cell lines, which are expected to improve the safety profile and facilitate the large-scale production of LAVs.

\section{Deletion of the virulence-associated gene(s)}

The precise deletion VAG of virulent strain to construct LAV candidates has become a critical approach to current ASF LAVs research. Compared with isolation of NA strains and attenuation of virulent strain by cell passage, individual or combined deletion of VAG can attenuate virulent ASFV isolate and induce protective immune responses against virulent parental virus challenge in pigs (Table 1). A recombinant LAV strain $\mathrm{BA} 71 \triangle \mathrm{CD} 2 \mathrm{v}$, lacking the $C D 2 v$ gene, can confer protection against homologous or heterologous ASFV infection [19]. A further study showed that $83.3 \%$ and $33.3 \%$ of the pigs immunized with $10^{6.0} \mathrm{PFU}$ BA71 $\triangle \mathrm{CD} 2$ survived when challenged with the genotypes XIX and IX ASFV, respectively [20]. These results confirmed that cross-protection is a multifactorial phenomenon that not only depends on sequence similarity [20]. The genotype II ASFV LAV strain ASFV-G- $\triangle 1177 \mathrm{~L}$, constructed by deleting the $1177 \mathrm{~L}$ gene from the virulent strain ASFV-G, has been proven safe and highly efficacious in challenge studies [21]. Further research has shown that the ASFVG- $\Delta$ I177L vaccine candidate can be administered by oronasal $(\mathrm{ON})$ route, which can achieve similar efficacy to intramuscular (IM) administration [22].

A Chinese research group generated a series of VAGsdeleted viruses based on the genotype II virulent strain ASFV HLJ/18, among which the mutant HLJ/18-7GD (lacking the MGF505-1R, MGF360-12L, MGF360-13L, MGF360-14L, MGF505-2R, MGF505-3R, and CD2v genes) was evaluated to be safe and protective in specificpathogen-free (SPF) pigs [23]. Subsequent field trials in fattening pigs, sows, and wild boar showed that HLJ/187 GD was genetically stable, unlikely to cause complications, and converted to virulence following vaccination. Our group showed that a genotype II ASFV mutant 
Table 1 Promising progress towards the development of live attenuated vaccines against African swine fever

\begin{tabular}{|c|c|c|c|c|c|c|}
\hline Parental strains & Genotypes & Attenuated strategies & LAV candidates & Protection & Production cells & References \\
\hline $\mathrm{NH} / \mathrm{P} 68$ & । & Naturally attenuated & NH/P68 & $\begin{array}{l}\text { Homologous strain (L60, } \\
\text { Arm07) }\end{array}$ & PBMs & [7] \\
\hline OURT88/3 & । & Naturally attenuated & OURT88/3 & $\begin{array}{l}\text { Homologous strain } \\
\text { (OURT88/1, Ug65) }\end{array}$ & BMs & [8] \\
\hline Lv17/WB/Rie1 & $\|$ & Naturally attenuated & Lv17/WB/Rie1 & $\begin{array}{l}\text { Homologous strain } \\
\text { (Armo7) }\end{array}$ & PBMs & {$[10,11]$} \\
\hline BA71 & । & DVAG (CD2v) & $\mathrm{BA} 71 \triangle \mathrm{CD} 2 \mathrm{v}$ & $\begin{array}{l}\text { Homologous and heterolo- } \\
\text { gous strain } \\
\text { (E75, Georgia 2007) }\end{array}$ & $\cos -1$ & [19] \\
\hline $\mathrm{HLJ} / 18$ & ॥ & $\begin{array}{l}\text { DVAG (MGF505-1R, MGF360- } \\
\text { 12L, MGF360-13L, MGF360- } \\
\text { 14L, MGF505-2R, MGF505-3R, } \\
\text { and CD2v) }\end{array}$ & $\mathrm{HLJ} / 18-7 \mathrm{GD}$ & $\begin{array}{l}\text { Homologous strain } \\
\text { (ASFV HLJ/18) }\end{array}$ & PAMs & [23] \\
\hline Georgia 2007 & $\|$ & DVAG (1177L) & ASFV-G- $\triangle 1177 \mathrm{~L}$ & $\begin{array}{l}\text { Homologous strain } \\
\text { (Georgia 2007) }\end{array}$ & PAMs & [21] \\
\hline ASFV-G- $\triangle 1177 \mathrm{~L}$ & $\|$ & DVAG (I177L) and cell passage & ASFV-G- $\Delta 1177 \mathrm{~L} / \Delta \mathrm{LVR}$ & $\begin{array}{l}\text { Homologous strain } \\
\text { (Georgia 2007) }\end{array}$ & PIPEC & {$[17]$} \\
\hline ASFV-SY18 & $\|$ & DVAG (CD2v and UK) & ASFV-SY18- $\triangle C D 2 v / U K$ & $\begin{array}{l}\text { Homologous strain } \\
\text { (ASFV-SY18) }\end{array}$ & PAMs & [24] \\
\hline ASFV-SY18 & $\|$ & DVAG (I226R) & $\mathrm{SY} 18 \Delta \mathrm{I} 226 \mathrm{R}$ & $\begin{array}{l}\text { Homologous strain } \\
\text { (ASFV-SY18) }\end{array}$ & PAMs & {$[25]$} \\
\hline Georgia 2010 & $\|$ & DVAG (A137R) & ASFV-G- $\triangle \mathrm{A} 137 \mathrm{R}$ & $\begin{array}{l}\text { Homologous strain } \\
\text { (Georgia 2010) }\end{array}$ & PAMs & {$[26]$} \\
\hline
\end{tabular}

DVAG deletion of virulence-associated genes; $P B M s$ porcine blood monocyte/macrophages; $B M s$ pig bone marrow cells; COS-1 monkey kidney tissue-derived cells; PAMs primary porcine alveolar macrophages; PIPEC Plum Island porcine epithelial cells (PIPEC), a porcine fetal kidney cell line engineered to express the bovine aV $\beta 6$ integrin; LAV Live attenuated vaccine

lacking the $C D 2 v$ and $U K$ genes was attenuated and elicited effective immune responses in pigs, providing complete protection against the challenge with the parental ASFV strain [24]. Although some new VAGs (e.g. I177L, $I 226 R$, and $A 137 R$ ) have been identified, the underlying mechanisms by which these genes affect virulence remain unclear $[21,25,26]$. The combined deletion of VAGs may over-attenuate ASFV and lead to a decrease or loss of protection $[27,28]$. In the next 3 to 5 years, strengthening the functional research of VAGs and identifying new VAGs will accelerate the rational engineering of ASF LAVs with improved safety profiles. However, the delicate balance between the attenuation and immunogenicity of ASFV is a major challenge for the development of ASF LAVs.

\section{Evaluation of LAV candidates}

After generating ASF LAV candidates through the above three strategies, it is necessary to conduct in vivo evaluation in biosafety level 3 (BSL-3) facilities to identify a safe and effective LAV candidate and then undergo a largescale clinical evaluation to determine whether the vaccine candidate can be commercialized (Fig. 1). The in vivo evaluation procedure for the virulence and safety of LAV candidates is to use different doses (e.g. $10^{2.0}-10^{7.0}$ $\left.\mathrm{HAD}_{50}\right)$ to inoculate pigs and observe the clinical signs including fever parameters, anorexia, recumbence, skin hemorrhage, or cyanosis, joint swelling, respiratory distress, and ocular discharge for 14-28 days [19-23]. Oropharyngeal, rectal excretion, and blood samples were collected at different days post-infection (dpi) to detect virus shedding, viremia, antibodies, and cellular immune response. Most of the general procedures for immunogenicity and protective efficacy evaluation via IM or ON route with $10^{2.0}-10^{7.0} \mathrm{HAD}_{50} \mathrm{LAV}$ candidate and challenge with a lethal dose of parental strain at 14-28 dpi, or after boost immunization with the same route on 14-28 dpi [29, 30]. According to current research, viremia after vaccination is a feature of most ASF LAVs, effectively protecting pigs against virulent ASFV challenges [17]. Few studies have used heterologous ASFV strain to evaluate cross-protection efficacy. Due to the multiple factors and the genetic diversity of ASFV that affect the evaluation results of LAV, it is necessary for global ASFV research experts to jointly draft and formulate guidelines for the trials of ASF LAVs, which will help to improve the reliability of the evaluation results.

\section{Major challenges impeding LAV development}

Currently, the commercialization prospect of ASF LAVs is not clear. In addition to the positive results, also have many safety issues, virus shedding, development of 


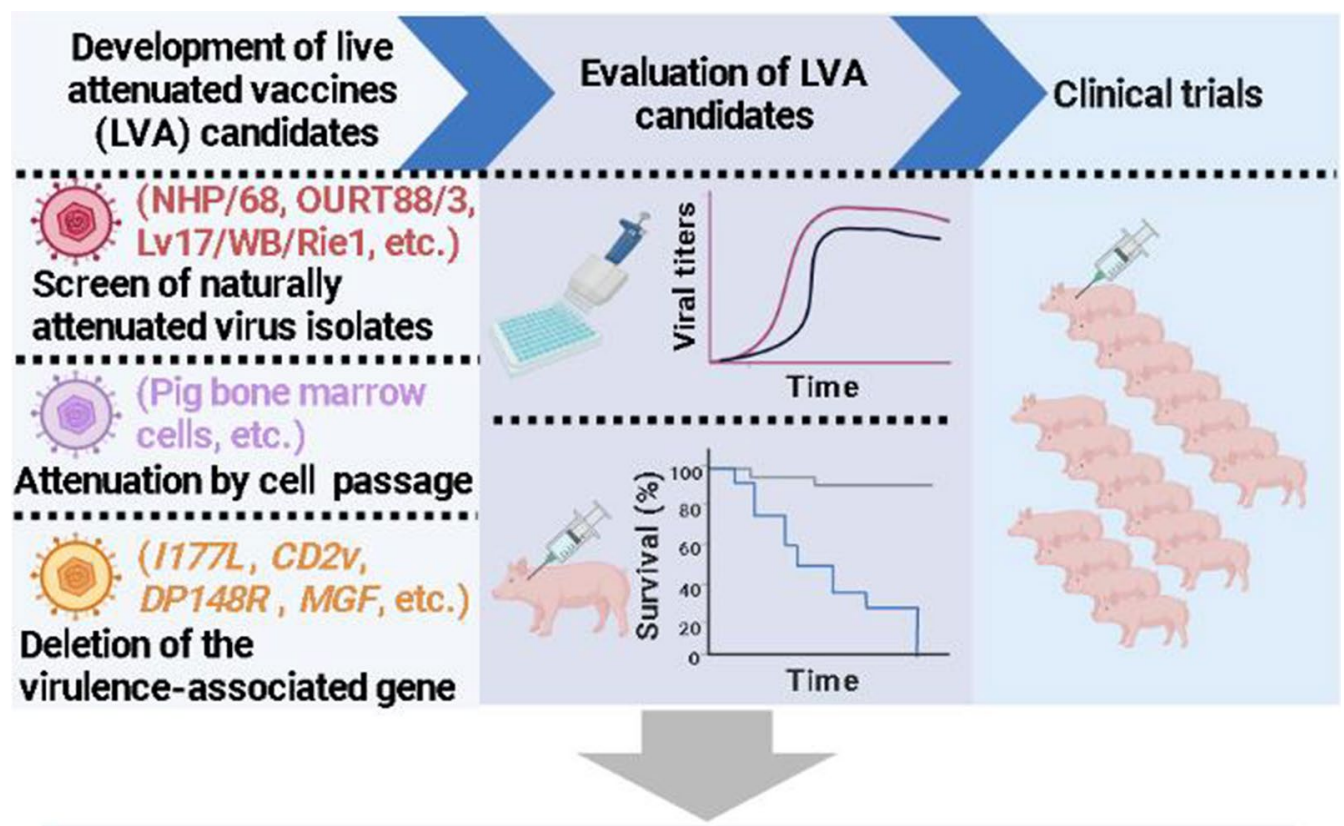

Major challenges impeding LVA development

1. Differentiation of infected from vaccinated animals

2. Selection of cell lines for vaccine production

3. Enhancement of cross-protection

Fig. 1 Development of live attenuated vaccines against African swine fever (created with BioRender.com)

post-vaccination complications, and insufficient protection of immunocompromised pigs. As discussed above, most ASF LAVs are evaluated through short immunization protocols, which is far from enough to evaluate the safety of LAVs. This is due to the insufficient market demand for ASF vaccines before 2018, and the evaluation tests of LAVs need to be carried out in BSL-3 facilities. Presently, the global epidemic of ASF has increased the market demand for the vaccines. The governments and enterprises have expanded funding to support ASF vaccine development. Therefore, it is necessary to evaluate the duration of protection of current LAV candidates [31]. This evaluation can provide reliable data support for clinical trials and help explore the molecular mechanism of vaccine-induced protective immunity and design a safe and effective vaccine against ASF.

The continuous passage of LAVs in pigs to assess the risks of conversion to virulence is very important for ensuring the safety of ASF LAVs. Infection with attenuated strains of different genetic backgrounds to assess the genetic recombination is also essential for evaluating the safety of ASF LAVs. These evaluations can determine whether ASF LAVs are safe enough to ensure that it can be used in the field. In addition, there are no adequate evaluations for the horizontal transmission of ASF LAVs and the risks of vertical transmission of sows after immunization. Therefore, it is still controversial whether the safety of candidate strains of ASF vaccines meets the requirements for application in the field.

When the safety and efficacy requirements are met, the first-generation ASF LAVs may be used under certain conditions, such as all-in and all-out fattening pig farms, pig farms in areas threatened by ASF outbreaks [32]. However, several problems still need to be solved for next-generation LAVs. Most LAVs are grown on PAMs, and no stable cell lines are available for manufacturing vaccines [29-32]. It has been reported that BA71 $\triangle C D 2 v$ can replicate in COS-1 cells, ASFV-G- $\Delta$ I177L can replicate in PIPEC cells after adaptation. However, the genome stability and immunogenicity maintenance of ASF LAVs after continuous passage in the abovementioned cells still need many assessments. ASF LAVs have limited cross-protective efficacy. It is still a considerable challenge to develop an LAV with cross-protective efficacy against genotypes I and II virulent ASFV strains circulating in Eurasia. 


\section{Detection technologies accompanying the gene-deleted ASF vaccines}

Developing a detection technology to differentiate infected from vaccinated-animal (DIVA) is essential for prevention, controlling, and eradicating ASF. There are two DIVA strategies for the ASF LAVs. One is to establish a multiplex real-time PCR targeting the $p 72$ gene of the wild-type ASFV strain and the deleted gene(s) of the ASF LAVs, respectively. The other is to detect antibodies induced by the deleted gene-encoded protein by enzyme-linked immunosorbent assay (ELISA). At present, based on CRISPR/Cas12a-based nucleic acid detection technology, recombinase polymerase amplification and gold nanoparticles technology have been reported for the rapid detection of ASFV. However, its commercial use still needs a particular time [33-36]. Currently widely used detection technology is the real-time PCR targeting the $p 72$ gene, which shows high sensitivity and specificity. NA ASFV strains could emerge after several years in the field, which induce inapparent or mild clinical signs with longer incubation time, thus the detection of antiASFV antibodies will be required for surveillance. In general, the development and commercialization of more sensitive, convenient, high-throughput, and easily accessible etiological and serological technologies are essential for monitoring and eradicating ASF.

\section{Conclusions}

A safe and effective vaccine and sensitive, specific, highthroughput, and readily available detection technologies are the basis for preventing, controlling, and eradicating ASF. They are also issues that the scientific community urgently needs to solve. In the near future, regardless of the success or failure of the scientific community to develop a safe and effective ASF vaccine, the control of ASF always requires prevention and control based on the principles of biosafety and rapid diagnosis.

\begin{abstract}
Abbreviations
ASF: African swine fever; ASFV: African swine fever virus; ORFs: Open reading frames; LAV: Live attenuated vaccine; NA: Naturally attenuated; non-HAD: Non-hemadsorbing; VAGs: Virulence-associated genes; ON: Oronasal; IM: Intramuscular; BLS-3: Biosafety level 3; DIVA: Differentiation of infected from vaccinated animals; DPI: Days post-infection; PAMs: Primary porcine alveolar macrophages; ELISA: Enzyme-linked immunosorbent assay.
\end{abstract}

\section{Acknowledgements}

We thank Dr. Yongfeng Li for his suggestions.

\section{Authors' contributions}

TW, RL, and YS wrote the manuscript. HJQ conceived and revised the manuscript. All authors read and approved the final manuscript.

\section{Funding}

This work was supported by the National Natural Science Foundation of China (Grant Nos. U20A2060 and 32072854).
Availability of data and materials

Not applicable.

\section{Declarations}

Ethics approval and consent to participate

Not applicable.

\section{Consent for publication \\ Not applicable.}

\section{Competing interests}

The authors declared that they have no competing interests.

\section{Author details}

${ }^{1}$ State Key Laboratory of Veterinary Biotechnology, Harbin Veterinary Research Institute, Chinese Academy of Agricultural Sciences, Harbin 150069, China.

${ }^{2}$ School of Life Science Engineering, Foshan University, Foshan 528231, China.

Received: 2 August 2021 Accepted: 11 November 2021

Published online: 24 December 2021

\section{References}

1. Alonso C, Borca M, Dixon L, Revilla Y, Rodriguez F, Escribano JM. ICTV virus taxonomy profile: Asfarviridae. J Gen Virol. 2018;99(5):613-4.

2. Montgomery RE. On a form of swine fever occurring in British East Africa (Kenya colony). J Comp Pathol. 1921;34:159-91.

3. Zhou X, Li N, Luo Y, Liu Y, Miao F, Chen T, et al. Emergence of African swine fever in China, 2018. Transbound Emerg Dis. 2018;65(6):1482-4.

4. Dixon LK, Stahl K, Jori F, Vial L, Pfeiffer DU. African swine fever epidemiology and control. Annu Rev Anim Biosci. 2020;8:221-46.

5. Wang T, Sun Y, Huang S, Qiu HJ. Multifaceted immune responses to African swine fever virus: implications for vaccine development. Vet Microbiol. 2020;249:108832

6. Teklue T, Sun Y, Abid M, Luo Y, Qiu HJ. Current status and evolving approaches to African swine fever vaccine development. Transbound Emerg Dis. 2020;67(2):529-42.

7. Leitão A, Cartaxeiro C, Coelho R, Cruz B, Parkhouse RME, Portugal FC, et al. The non-haemadsorbing African swine fever virus isolate ASFV/NH/P68 provides a model for defining the protective anti-virus immune response. J Gen Virol. 2001;82:513-23.

8. Boinas FS, Hutchings GH, Dixon LK, Wilkinson PJ. Characterization of pathogenic and non-pathogenic African swine fever virus isolates from Ornithodoros erraticus inhabiting pig premises in Portugal. J Gen Virol. 2004;85:2177-87.

9. Zani L, Forth JH, Forth L, Nurmoja I, Leidenberger S, Henke J, et al. Deletion at the $5^{\prime}$-end of Estonian ASFV strains associated with an attenuated phenotype. Sci Rep. 2018;8(1):6510.

10. Gallardo C, Soler A, Rodze I, Nieto R, Cano-Gómez C, Fernandez-Pinero J, et al. Attenuated and non-haemadsorbing (non-HAD) genotype II African swine fever virus (ASFV) isolated in Europe, Latvia 2017. Transbound Emerg Dis. 2019;66(3):1399-404.

11. Barasona JA, Gallardo C, Cadenas-Fernández E, Jurado C, Rivera B, Rodríguez-Bertos A, et al. First oral vaccination of Eurasian wild boar against African swine fever virus genotype II. Front Vet Sci. 2019;6:137.

12. Sun E, Zhang Z, Wang Z, He X, Zhang X, Wang L, et al. Emergence and prevalence of naturally occurring lower virulent African swine fever viruses in domestic pigs in China in 2020. Sci China Life Sci. 2021;64(5):752-65.

13. Manso RJ, Nunes Petisca JL, Lopes Frazao F, Sobral M. Vaccination contre la peste porcine Africaine. Bulletin de L'Office International des Épizooties. 1963;60:921-37.

14. Sánchez BC. Modificatión del virus de la peste porcina Africana en cultivos celulares. Bulletin de l'Office International des Epizooties. 1963;60:901-19.

15. Balysheva VI, Prudnikova EY, Galnbek TV, Balyshev VM. Immunological properties of attenuated variants of African swine fever virus isolated in the Russian Federation. Russ Agric Sci. 2015;41(2):178-82. 
16. Krug PW, Holinka LG, O'Donnell V, Reese B, Sanford B, Fernandez-Sainz I, et al. The progressive adaptation of a Georgian isolate of African swine fever virus to Vero cells leads to a gradual attenuation of virulence in swine corresponding to major modifications of the viral genome. J Virol. 2015;89(4):2324-32

17. Borca MV, Rai A, Ramirez-Medina E, Silva E, Velazquez-Salinas L, Vuono $E$, et al. A cell culture-adapted vaccine virus against the current African swine fever virus pandemic strain. J Virol. 2021;95(14):e0012321.

18. Wang T, Wang L, Han Y, Pan L, Yang J, Sun M, et al. Adaptation of African swine fever virus to HEK293T cells. Transbound Emerg Dis. 2021;68(5):2853-66.

19. Monteagudo PL, Lacasta A, López E, Bosch L, Collado J, Pina-Pedrero S, et al. BA71 $\triangle C D 2$ : a new recombinant live attenuated African swine fever virus with Cross-Protective capabilities. J Virol. 2017;91 (21):e01058-17.

20. Lopez E, van Heerden J, Bosch-Camós L, Accensi F, Navas MJ, LópezMonteagudo P, et al. Live attenuated African swine fever viruses as ideal tools to dissect the mechanisms involved in Cross-protection. Viruses. 2020;12(12):1474.

21. Borca MV, Ramirez-Medina E, Silva E, Vuono E, Rai A, Pruitt S, et al. Development of a highly effective African swine fever virus vaccine by deletion of the $1177 \mathrm{~L}$ gene results in sterile immunity against the current Epidemic Eurasia strain. J Virol. 2020;94(7):e02017-19.

22. Borca MV, Ramirez-Medina E, Silva E, Vuono E, Rai A, Pruitt S, et al. ASFV$\mathrm{G}-\Delta 1177 \mathrm{~L}$ as an effective oral nasal vaccine against the Eurasia strain of Africa swine fever. Viruses. 2021;13(5):765.

23. Chen W, Zhao D, He X, Liu R, Wang Z, Zhang X, et al. A seven-genedeleted African swine fever virus is safe and effective as a live attenuated vaccine in pigs. Sci China Life Sci. 2020;63(5):623-34.

24. Teklue T, Wang T, Luo Y, Hu R, Sun Y, Qiu HJ. Generation and evaluation of an African swine fever virus mutant with deletion of the CD2v and UK genes. Vaccines. 2020;8(4):763.

25. Zhang Y, Ke J, Zhang J, Yang J, Yue H, Zhou X, et al. ASFV bearing an I226R gene-deletion elicits a robust immunity in pigs to African swine fever. J Virol. 2021:JVI0119921. https://doi.org/10.1128/JVI.01199-21.

26. Gladue DP, Ramirez-Medina E, Vuono E, Silva E, Rai A, Pruitt S, et al. Deletion of A137R gene from the pandemic strain of African swine fever virus is attenuated and offers protection against virulent pandemic virus. J Virol. 2021:JVI0113921. https://doi.org/10.1128/JVI.01139-21.

27. Gladue DP, O'Donnell V, Ramirez-Medina E, Rai A, Pruitt S, Vuono EA, et al. Deletion of CD2-like (CD2v) and C-Type lectin-like (EP153R) genes from African swine fever virus Georgia- $\triangle 9 \mathrm{GL}$ abrogates its effectiveness as an experimental vaccine. Viruses. 2020;12(10):1185.

28. Lopez E, Bosch-Camós L, Ramirez-Medina E, Vuono E, Navas MJ, Muñoz $M$, et al. Deletion mutants of the attenuated recombinant ASF virus, BA71 $\triangle C D 2$, show decreased vaccine efficacy. Viruses. 2021;13(9):1678.

29. Sereda AD, Balyshev VM, Kazakova AS, Imatdinov AR, Kolbasov DV. Protective properties of attenuated strains of African swine fever virus belonging to seroimmunotypes I-VIII. Pathogens. 2020;9(4):274.

30. Turlewicz-Podbielska H, Kuriga A, Niemyjski R, Tarasiuk G, Pomorska-Mól M. African swine fever virus as a difficult opponent in the fight for a vaccine-current data. Viruses. 2021;13(7):1212.

31. Sánchez-Cordón PJ, Jabbar T, Chapman D, Dixon LK, Montoya M. Absence of long-term protection in domestic pigs immunized with attenuated African swine fever virus isolate OURT88/3 or Benin $\triangle M G F$ correlates with increased levels of regulatory T Cells and interleukin-10. J Virol. 2020;94(14):e00350-e420.

32. Rock DL. Thoughts on African swine fever vaccines. Viruses. 2021;13(5):943.

33. He Q, Yu D, Bao M, Korensky G, Chen J, Shin M, et al. High-throughput and all-solution phase African swine fever virus (ASFV) detection using CRISPR-Cas 12a and fluorescence based point-of-care system. Biosens Bioelectron. 2020;154:112068.

34. Tao D, Liu J, Nie X, Xu B, Tran-Thi TN, Niu L, et al. Application of CRISPRCas 12a enhanced fluorescence assay coupled with nucleic acid amplification for the sensitive detection of African swine fever virus. ACS Synth Biol. 2020;9(9):2339-50.

35. Fan X, Li L, Zhao Y, Liu Y, Liu C, Wang Q, et al. Clinical validation of two recombinase-based isothermal amplification assays (RPA/RAA) for the rapid detection of African swine fever virus. Front Microbiol. 2020;11:1696.
36. Qiu Z, Li Z, Yan Q, Li Y, Xiong W, Wu K, et al. Development of diagnostic tests provides technical support for the control of African swine fever. Vaccines. 2021;9(4):343.
Ready to submit your research? Choose BMC and benefit from:

- fast, convenient online submission

- thorough peer review by experienced researchers in your field

- rapid publication on acceptance

- support for research data, including large and complex data types

- gold Open Access which fosters wider collaboration and increased citations

- maximum visibility for your research: over $100 \mathrm{M}$ website views per year

At BMC, research is always in progress.

Learn more biomedcentral.com/submissions 